\title{
NGC 4569: Recent evidence for a past ram pressure stripping event ${ }^{\star}$
}

\author{
B. Vollmer ${ }^{1,2}$, C. Balkowski ${ }^{3}$, V. Cayatte ${ }^{3}$, W. van Driel $^{3}$, and W. Huchtmeier ${ }^{2}$ \\ ${ }^{1}$ CDS, Observatoire astronomique de Strasbourg, UMR 7550, 11 rue de l'université, 67000 Strasbourg, France \\ 2 Max-Planck-Institut für Radioastronomie, Auf dem Hügel 69, 53121 Bonn, Germany \\ 3 Observatoire de Paris, Section de Meudon, GEPI, CNRS UMR 8111 and Université Paris 7, 5 place Jules Janssen, \\ 92195 Meudon Cedex, France
}

Received 21 October 2003 / Accepted 14 January 2004

\begin{abstract}
Deep 21-cm H I line observations of the Virgo cluster spiral galaxy NGC 4569 have been obtained with the VLA in its D configuration and with the Effelsberg 100-m telescope. A low surface density arm was discovered in the west of the galaxy, whose velocity field is distinct from that of the overall disk rotation. The observed gas distribution, velocity field and velocity dispersion are compared to snapshots of dynamical simulations that include the effects of ram pressure. Two different scenarios were explored: (i) ongoing stripping and (ii) a major stripping event that took place about $300 \mathrm{Myr}$ ago. It is concluded that only the post-stripping scenario can reproduce the main observed characteristics of NGC 4569. It is not possible to determine if the gas disk of NGC 4569 had already been truncated before it underwent the ram pressure event that lead to its observed H I deficiency.
\end{abstract}

Key words. galaxies: individual: NGC 4569 - galaxies: interactions - galaxies: ISM - galaxies: kinematics and dynamics

\section{Introduction}

The spiral galaxy NGC 4569 is a very peculiar member of the Virgo cluster. Its very large size $\left(D_{25}=9.5\right.$, or $47 \mathrm{kpc}$ at the assumed Virgo cluster distance of $17 \mathrm{Mpc}$ ) compared to other cluster members and negative radial velocity $\left(v_{\mathrm{r}}=-235 \mathrm{~km} \mathrm{~s}^{-1}\right.$ with respect to the cluster mean velocity of $\sim 1100 \mathrm{~km} \mathrm{~s}^{-1}$ ) have lead to some doubt about its cluster membership, despite its close projected distance to the cluster center $\left(d=1.7^{\circ}\right)$. Van den Bergh (1976) classified this very red galaxy as anemic due to its low arm-interarm contrast on optical images. H I line synthesis observations (Warmels 1988; Cayatte et al. 1990) showed that it has lost more than $90 \%$ of its H I gas and that its H I distribution is heavily truncated. NGC 4569 is thus an exceptional galaxy, it is one of the largest and most H I deficient galaxies in the Virgo cluster (Solanes et al. 2001). Since field galaxies are generally not $\mathrm{HI}$ deficient, this is proof that NGC 4569 is a Virgo cluster member whose atomic gas has been most probably stripped due to its rapid motion through the hot intracluster medium (ICM). Nevertheless, it is not clear when and where NGC 4569 lost its gas.

A possible hint to its stripping history may be derived from X-ray observations. The analysis of ROSAT observations

\footnotetext{
Send offprint requests to: $\mathrm{B}$. Vollmer, e-mail: bvollmer@astro.u-strasbg.fr

* Movies are available in electronic form at http://www . edpsciences.org
}

(Tschöke et al. 2001) showed that there is no soft emission $(0.1-0.4 \mathrm{keV})$ from the northern half of the disk, whereas it is pronounced in the southern disk. This is consistent with a scenario where NGC 4569 is moving to the north-east, which causes ram pressure to push the hot ISM in the galaxy towards the south-west.

In addition, NGC 4569 harbors a bright, compact optical nucleus that, according to Keel (1996), is due to a starburst dominated by supergiant stars. Tschöke et al. (2001) suggest that a part of the observed extended X-ray emission is due to an outflow of hot ISM from the nucleus. The presence of such a feature in the west and its absence in the east is also consistent with galaxy motion towards the north-east. In the following we will not discuss the possiblity of nuclear outflow, since it expands vertically to the disk and does not affect the dynamics of the atomic gas, which is mainly located in the disk.

In the present paper we report on new deep VLA $21 \mathrm{~cm}$ H I observations of NGC 4569, which reveal a new and unexpected feature - a kinematically perturbed, low surface brightness arm - and we present models of the gas distribution and velocity field based on numerical simulations that include the effect of ram pressure stripping. This enables us to date the latest ISM-ICM interaction that led to the large H I deficiency of NGC 4569. The present work is part of a series of articles where we aim to understand the physics of ICM-ISM interactions (Vollmer et al. 1999, 2001; Vollmer 2003; Vollmer \& Huchtmeier 2003). 
Table 1. Physical parameters of NGC 4569.

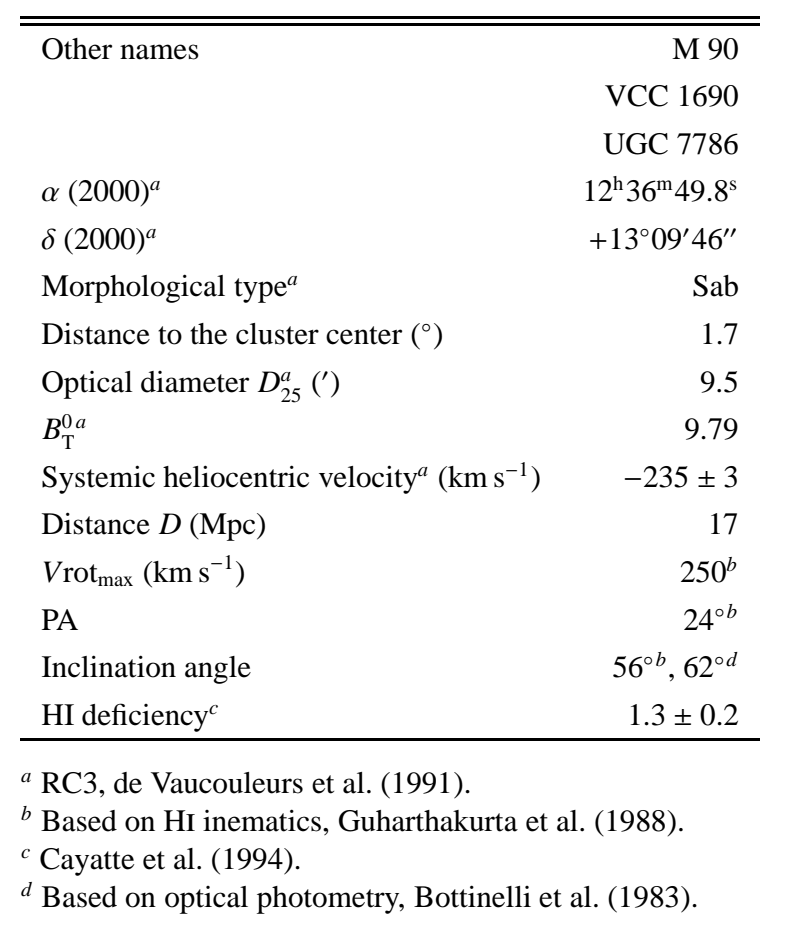

This article is organized in the following way: the observations and their results are described in Sects. 2 and 3, the dynamical model is presented in Sect. 4, our model snapshots are compared with the observations in Sect. 5 and discussed in Sect. 6. The conclusions are given in Sect. 7.

\section{Observations}

\subsection{VLA}

The $21 \mathrm{~cm}$ line observations were made with the Very Large Array (VLA); for a description see Napier et al. (1983). The field was centered on NGC 4569 (see Table 1). We observed on November 23 and 29, 2001 for a total of $290 \mathrm{~min}$ with the D configuration. The observed bandwidth of $3 \mathrm{MHz}$ was divided into 63 velocity channels of $48.8 \mathrm{kHz}\left(\sim 10 \mathrm{~km} \mathrm{~s}^{-1}\right)$ each. The velocity channels are centered on $v_{\text {hel }} \sim-235 \mathrm{~km} \mathrm{~s}^{-1}$. The data were calibrated using the standard VLA data reduction program package (AIPS). All strong continuum sources were subtracted directly in the UV plane using a linear interpolation of the UV data points of the first and last 10 channels. The final rms noise per $10 \mathrm{~km} \mathrm{~s}^{-1}$ wide channel at a smoothed spatial resolution of $52^{\prime \prime} \times 40^{\prime \prime}$ is $0.6 \mathrm{mJy} /$ beam, which is close to the expected value. We combined the UV data directly with the C array data of Cayatte et al. (1990), which were processed in the same way. The same weight was attributed to both data sets. Since the $\mathrm{C}$ array data have a channel width of $20 \mathrm{~km} \mathrm{~s}^{-1}$, we binned every 2 channels of our D array data before joining it with the $\mathrm{C}$ array data. The velocity offset between the two data sets is $\Delta v=4 \mathrm{~km} \mathrm{~s}^{-1}$. The resulting image of the joined UV data was CLEANed with a $39^{\prime \prime} \times 31^{\prime \prime} F W H M$ beam. We had a final rms noise of $0.5 \mathrm{mJy} /$ beam in one $20 \mathrm{~km} \mathrm{~s}^{-1}$ wide
Table 2. Integration times and rms.

\begin{tabular}{lcccccc}
\hline \hline Position & $\mathrm{C}$ & $\mathrm{NW}$ & $\mathrm{W}$ & $\mathrm{SW}$ & $\mathrm{SE}$ & $\mathrm{NE}$ \\
\hline$\Delta t(\mathrm{~min})$ & 120 & 120 & 120 & 120 & 120 & 120 \\
\hline $\mathrm{rms}(\mathrm{mJy})$ & 1.3 & 1.1 & 2.0 & 1.6 & 1.6 & 1.5 \\
\hline
\end{tabular}

channel. A flux of $1 \mathrm{mJy} \mathrm{km} \mathrm{s}^{-1}$ /beam corresponds to an H I column density of $10^{18} \mathrm{~cm}^{-2}$.

\subsection{Effelsberg}

On March 14-21, 2002, we performed 21-cm line observations at 6 different positions centered on the systemic velocity of NGC 4569 with a bandwidth of $12.5 \mathrm{MHz}$. The two-channel receiver had a system noise of $\sim 30 \mathrm{~K}$. The 1024 channel autocorrelator was split into four banks with 256 channels each, yielding a channel separation of $\sim 10 \mathrm{~km} \mathrm{~s}^{-1}$. We further binned the channels to obtain a final channel separation of $\sim 20 \mathrm{~km} \mathrm{~s}^{-1}$ as for the VLA data. The galaxy's central position and four positions at a distance of one beam width $\left(9.3^{\prime}\right)$ to the NW, SW, SE, and NE from the galaxy center were observed in on-off mode ( 5 min on source, 5 min off source). In addition, we observed a sixth position 6.5 west of the galaxy center. Care was taken to avoid other Virgo cluster galaxies with velocities within our bandwidth in all observations. We used 3C 286 for pointing and flux calibration. The observation time was 120 min per position. The resulting noise (Table 2 ) is partly determined by small amplitude interferences, but it is close to the theoretical noise of $2 \mathrm{mJy}$ per hour of integration: on average $1.5 \mathrm{mJy}$ (varying from 1.1 to $2.0 \mathrm{mJy}$ ).

\section{Results}

\subsection{The VLA data}

The channel maps are presented in Fig. 1. Although two channels $\left(v=-30 \mathrm{~km} \mathrm{~s}^{-1}\right.$ and $\left.-10 \mathrm{~km} \mathrm{~s}^{-1}\right)$ are contaminated by Galactic HI, the HI of NGC 4569 at these velocities can still be recognized and isolated from it. The galactic rotation of NGC 4569 is clearly visible. The most interesting feature appears in the velocity range between $-400 \mathrm{~km} \mathrm{~s}^{-1}$ and $-276 \mathrm{~km} \mathrm{~s}^{-1}$, where a detached emission region can be seen in the south-west part of NGC 4569, which shows rotation.

We have produced an integrated H I spectrum of NGC 4569 by adding all signals exceeding the $3 \sigma$ level in all channels that contain line emission, inside an area around NGC 4569 small enough not to pick up Galactic HI. Figure 2 shows the integrated VLA spectrum together with the Effelsberg 100-m data of the central pointing. The Effelsberg beam of 9.3' includes the the whole disk HI emission. The Galactic foreground is responsible for the large peak in the Effelsberg spectrum at $\sim-220 \mathrm{~km} \mathrm{~s}^{-1}$ relative to the systemic velocity, whose width $(F W H M)$ is $\Delta v \sim 50 \mathrm{~km} \mathrm{~s}^{-1}$. The total flux and peak flux density of Cayatte et al. (1990) are $\sim 20 \%$ higher than our VLA ( $\mathrm{C}$ and $\mathrm{D}$ array) values, which is within the calibration accuracy. Both of our spectra agree reasonably well, and both peaks 


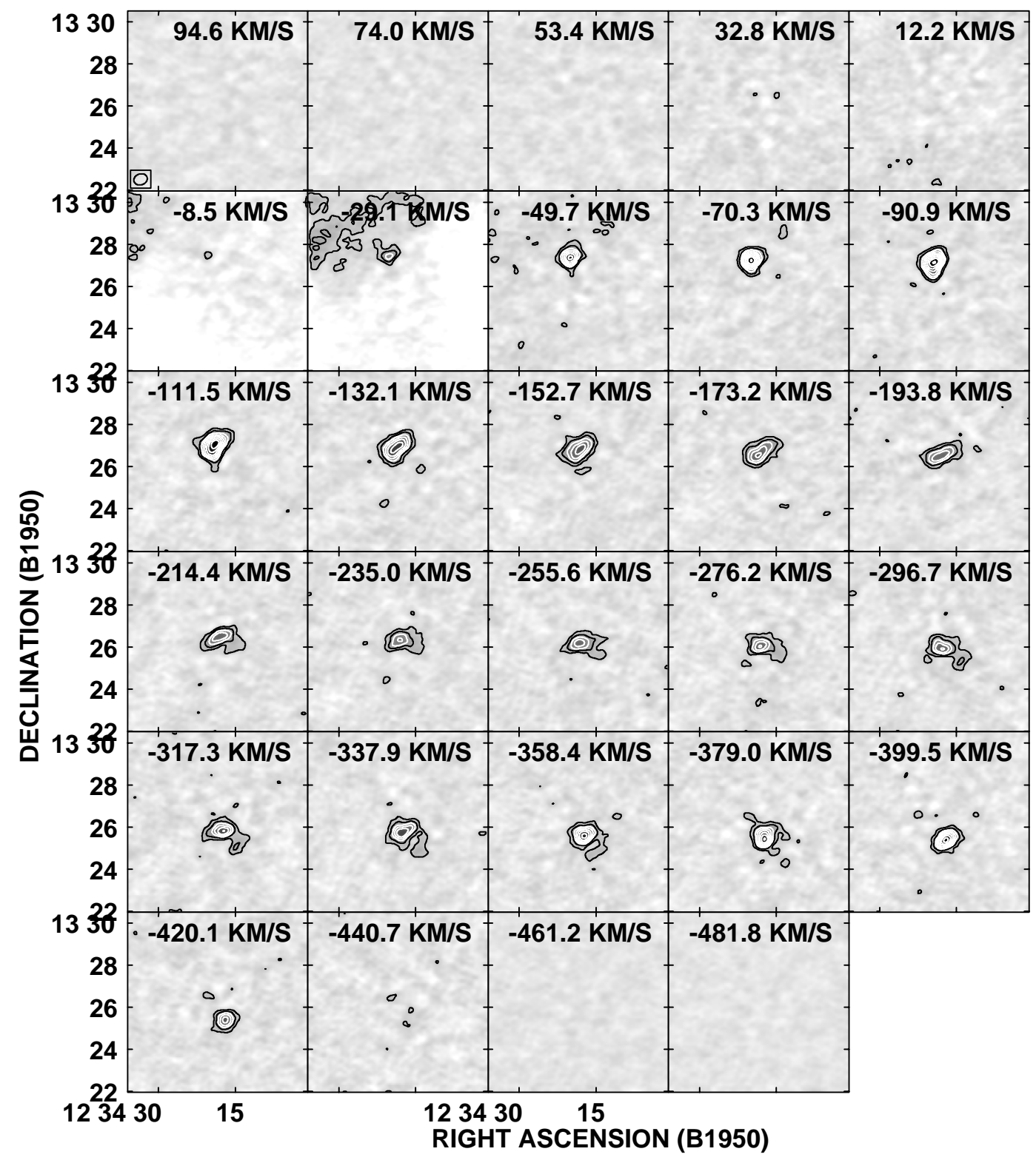

Fig. 1. VLA H I channel maps of NGC 4569. The central velocity of each channel is indicated in the upper part of each panel. The HPBW $\left(31^{\prime \prime} \times 39^{\prime \prime}\right)$ is plotted in the lower left corner of the first channel. The contours are in steps of $3 \sigma=1.5 \mathrm{mJy} / \mathrm{beam}$ beginning at $1.5 \mathrm{mJy} / \mathrm{beam}$.

of the double horned shaped spectrum have about the same peak flux density. We derive a linewidth at $20 \% / 50 \%$ of the peak flux of $W_{20}=390 \mathrm{~km} \mathrm{~s}^{-1} / W_{50}=350 \mathrm{~km} \mathrm{~s}^{-1}$ and a total flux of $8.85 \mathrm{Jy} \mathrm{km} \mathrm{s}^{-1}$, which corresponds to a total H I mass of $6 \times 10^{8} M_{\odot}$ assuming a distance of $17 \mathrm{Mpc}$.

The first three moment maps (column density, velocity field, and velocity dispersion) can be seen in Fig. 3. For the determination of all moment maps the data were clipped at a level of $3 \sigma=1.5 \mathrm{mJy} / \mathrm{beam}$ in each channel containing $\mathrm{HI}$ emission. The H I distribution consists mainly of three maxima: at the center, to the north and to the south. The southern maximum lies west of the major axis. The $\mathrm{H}$ I is thus not uniformly distributed within the disk, but might be distributed along spiral arms as is the case for NGC 4579 (Cayatte et al. 1990) or NGC 4548 (Vollmer et al. 1999). We observe a low surface density arm to the west, which was already visible on the channel maps. This arm can also be seen on the $\mathrm{H} \alpha$ image presented in Tschöke et al. (2001) and the blue optical image shown in Fig. 4.

The velocity field of NGC 4569 is quite symmetric within the disk. The roation curve rises on both sides with a maximum of $250 \mathrm{~km} \mathrm{~s}^{-1}$ assuming the kinematic inclination angle of $i=56^{\circ}$ (Guharthakurta et al. 1988) or $230 \mathrm{~km} \mathrm{~s}^{-1}$ assuming the inclination based on the optical morphology $i=62^{\circ}$ (Bottinelli et al. 1983). The western, low surface density arm is kinematically distinct from the disk gas. This arm shows rotation, but has systematically higher velocities than the disk gas.

As expected, the velocity dispersion shows a maximum in the galaxy center, due to rotation and beam smearing. The absolute maximum is located $\sim 10^{\prime \prime}$ to the north-east of the nucleus and has a velocity dispersion greater than $80 \mathrm{~km} \mathrm{~s}^{-1}$, a second 


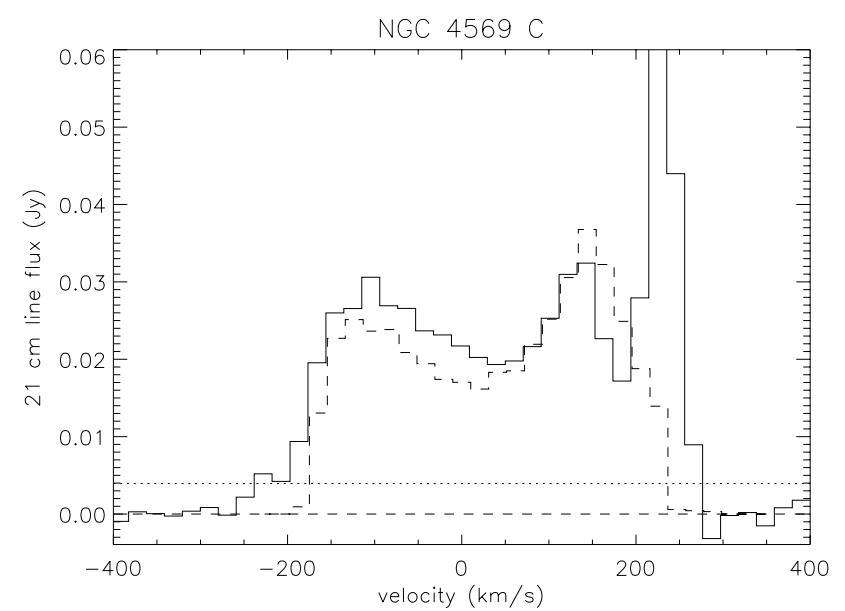

Fig. 2. Solid line: Effelsberg spectrum of the central position. Dashed line: integrated VLA spectrum. Dotted line: $3 \sigma$ noise level of the Effelsberg spectrum. The radial velocities are relative to the systemic velocity of NGC 4569.

more extended maximum is observed at the inner edge of the western, low surface density arm south of the nucleus.

\subsection{The Effelsberg data}

For the 4 off-center Effelsberg positions we have synthesized spectra from the VLA data using the area defined by the pointing positions and the (9.3) Effelsberg HPBW. In this way we take advantage of the spatial HI distribution of the VLA data (see Vollmer \& Huchtmeier 2003). Figure 5 shows the Effelsberg spectra of these positions (solid lines) together with the synthesized VLA spectra. Since the VLA data cube shows only H I gas located in the disk (see Cayatte et al. 1990), any emission excess in the off-center Effelsberg spectra must be due to gas located outside the disk. No such excess is seen and an upper mass limit $\sim 10^{7} M_{\odot}$ can be set for the $\mathrm{H}$ I outside the disk, corresponding to a signal of $5 \sigma$ in a $20 \mathrm{~km} \mathrm{~s}^{-1}$ channel with the an rms of $\sigma=1.6 \mathrm{mJy}$. The absence of atomic gas far away from the galaxy (>20 kpc), as was found for NGC 4388 (Vollmer \& Huchtmeier 2003), is an important constraint on dynamic stripping models.

\section{Simulations}

We have adopted a model where the ISM is simulated as a collisional component, i.e. as discrete particles that possess a mass and a radius and which can have inelastic collisions (sticky paticles). Since the ISM is a turbulent and fractal medium (see e.g. Elmegreen \& Falgarone 1996), it is neither continuous nor discrete. The volume filling factor of the different phases is smaller than one. The warm neutral and ionized gas fill about $50 \%$ of the volume, whereas cold neutral gas has a volume filling factor of only $2 \%$ (Boulares \& Cox 1990). It is not clear how this fraction changes when an external pressure is applied. In contrast to smoothed particles hydrodynamics (SPH), which is a quasi continuous approach and where the particles cannot penetrate each other, our approach allows a finite penetration length, which is given by the mass-radius relation of the
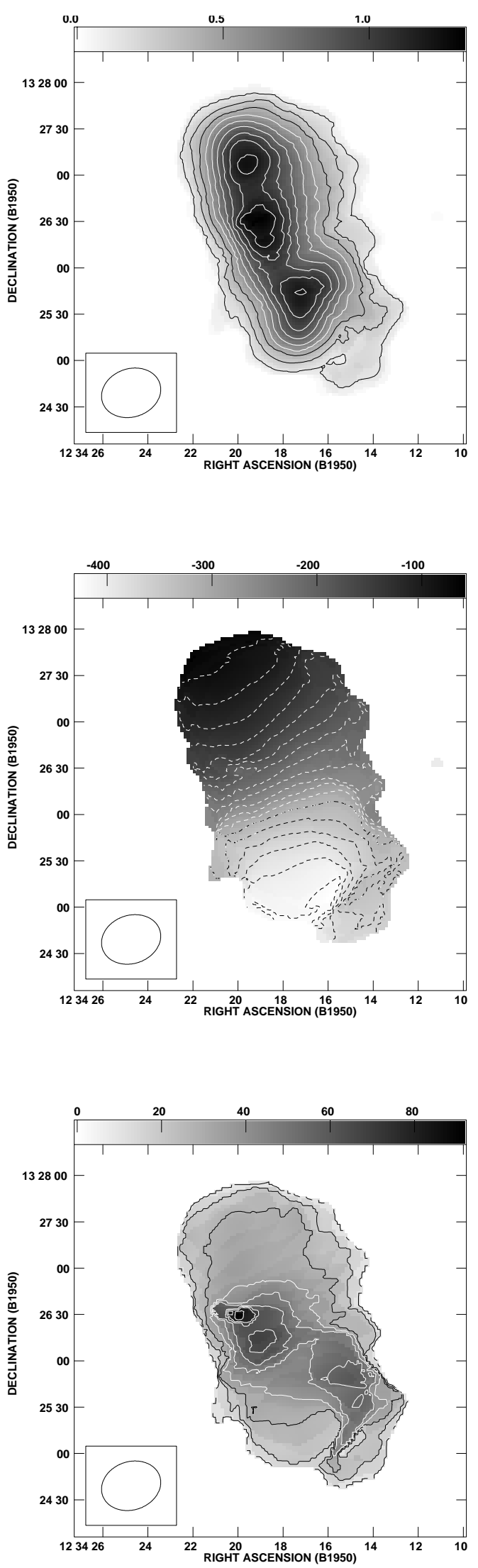

Fig. 3. H I moment maps of NGC 4569. Upper panel: column density. The contour steps are from 1 to $9 \times 1.3 \times 10^{20} \mathrm{~cm}^{-2}$. Middle panel: velocity field. Contour levels are from -440 to $-40 \mathrm{~km} \mathrm{~s}^{-1}$ in steps of $20 \mathrm{~km} \mathrm{~s}^{-1}$. Lower panel: velocity dispersion. Contour levels are from 1 to $9 \times 8.3 \mathrm{~km} \mathrm{~s}^{-1}$. 


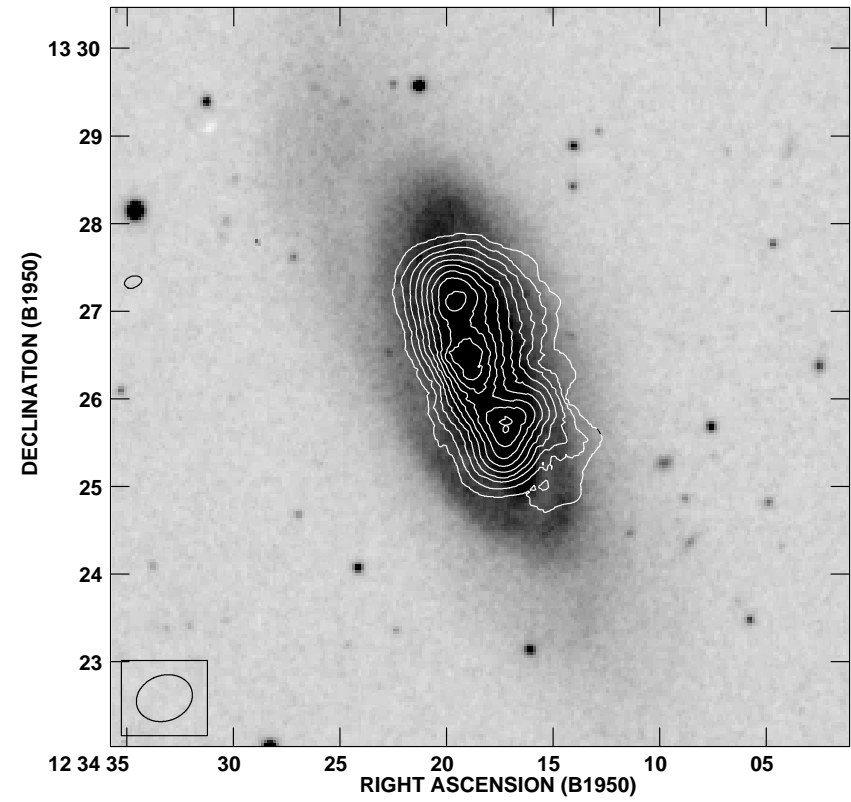

Fig. 4. Contours: H I column density. The contour levels are the same as in Fig. 3. Greyscale: B band DSS image. The contrast is chosen such that the whole disk extent becomes visible.

particles. Both methods have their advantages and their limits. The advantage of our approach is that ram pressure can be included easily as an additional acceleration on particles that are not protected by other particles (see Vollmer et al. 2001). In this way we avoid the problem of treating the large density contrast between the $\operatorname{ICM}\left(n \sim 10^{-4} \mathrm{~cm}^{-3}\right)$ and the $\operatorname{ISM}\left(n>1 \mathrm{~cm}^{-3}\right)$ of the galaxy.

Since the model is described in detail in Vollmer et al. (2001), we will summarize only its main features. The $N$-body code consists of two components: a non-collisional component that simulates the stellar bulge/disk and the dark halo, and a collisional component that simulates the ISM. The 20000 particles of the collisional component represent gas cloud complexes that are evolving in the gravitational potential of the galaxy.

The total assumed gas mass is $M_{\text {gas }}^{\text {tot }}=6.2 \times 10^{9} M_{\odot}$, which corresponds to the total neutral gas mass before stripping, i.e. to an H I deficiency of 1.0, which is defined as the logarithm of the ratio between the $\mathrm{H}$ I content of a field galaxy of same morphological type and diameter and the observed H I mass. To each particle a radius is attributed depending on its mass. During the disk evolution the particles can have inelastic collisions, the outcome of which (coalescence, mass exchange, or fragmentation) is simplified following Wiegel (1994). This results in an effective gas viscosity in the disk.

As the galaxy moves through the ICM, its clouds are accelerated by ram pressure. Within the galaxy's inertial system its clouds are exposed to a wind coming from a direction opposite to that of the galaxy's motion through the ICM. The temporal ram pressure profile has the form of a Lorentzian, which is realistic for galaxies on highly eccentric orbits within the Virgo cluster (Vollmer et al. 2001). The effect of ram pressure on the clouds is simulated by an additional force on the clouds
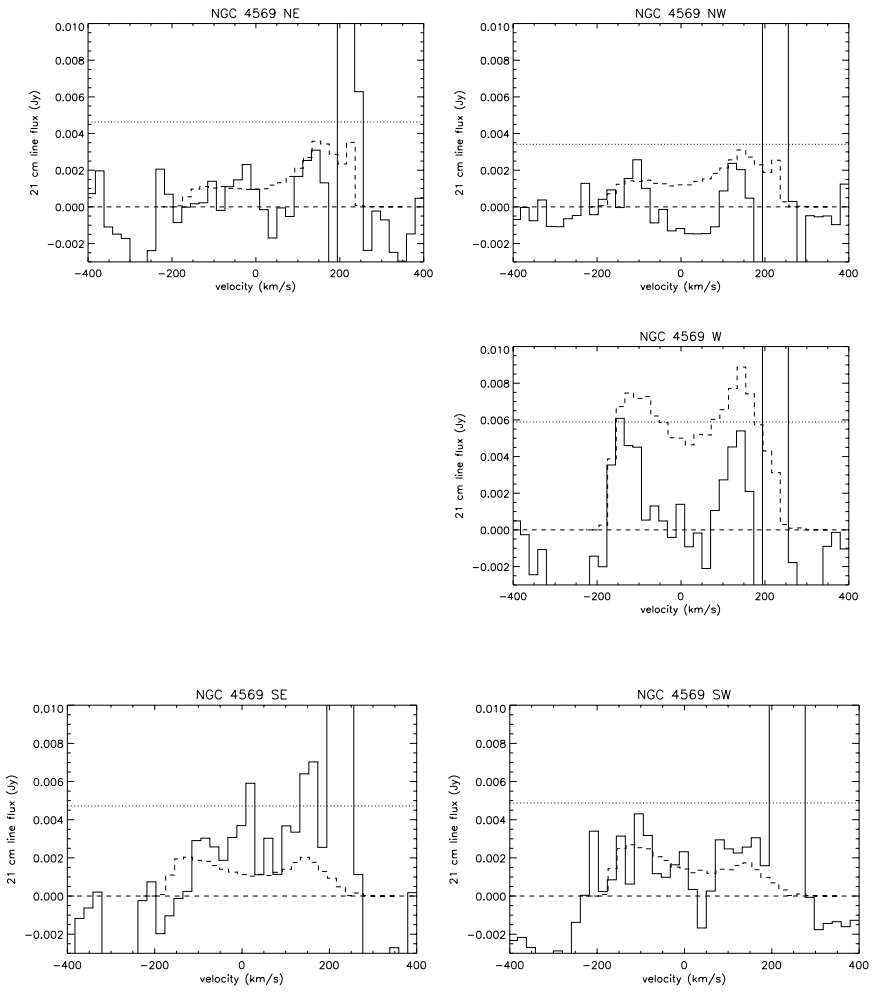

Fig. 5. Solid lines: Effelsberg 100-m spectra of the five off-center positions. Their locations with respect to the galaxy center are marked on top of each panel. Dashed line: synthesized VLA spectra, which only show H I disk emission. Dotted line: $3 \sigma$ noise levels of the Effelsberg spectra. Radial velocities are given relative to the systemic velocity of NGC 4569.

Table 3. Total mass, number of particles $N$, particle mass $M$, and smoothing length $l$ for the different galactic components.

\begin{tabular}{llcll}
\hline \hline component & $M_{\mathrm{tot}}\left(M_{\odot}\right)$ & $N$ & $M\left(M_{\odot}\right)$ & $l(\mathrm{pc})$ \\
\hline Halo & $2.4 \times 10^{11}$ & 16384 & $1.4 \times 10^{7}$ & 1200 \\
Bulge & $8.2 \times 10^{9}$ & 16384 & $5.0 \times 10^{5}$ & 180 \\
Disk & $4.1 \times 10^{10}$ & 16384 & $2.5 \times 10^{6}$ & 240 \\
\hline
\end{tabular}

in the wind direction. Only clouds that are not protected by other clouds from the wind are affected.

The non-collisional component consists of 49125 particles, which simulate the galactic halo, bulge, and disk. The characteristics of the different galactic components are shown in Table 3 . The resulting rotation velocity is $\sim 220 \mathrm{~km} \mathrm{~s}^{-1}$ and the rotation curve is flat.

The particle trajectories are integrated using an adaptive timestep for each particle. This method is described in Springel et al. (2001). The following criterion for an individual timestep is applied:

$\Delta t_{\mathrm{i}}=\frac{20 \mathrm{~km} \mathrm{~s}^{-1}}{a_{i}}$,

where $a_{i}$ is the acceleration of the particle $i$. The minimum value of $t_{i}$ defines the global timestep used for the Burlisch-Stoer integrator that integrates the collisional component. 
Table 4. Model parameters for time-dependent ram pressure stripping.

\begin{tabular}{lr}
\hline \hline Maximum ram pressure $\left(\mathrm{cm}^{-3}\left(\mathrm{~km} \mathrm{~s}^{-1}\right)^{2}\right)$ & 4000 \\
Inclination angle between orbital and disk plane & $35^{\circ}$ \\
Final HI deficiency & 1.0 \\
\hline
\end{tabular}

The galaxy is on an eccentric orbit within the cluster. The temporal ram pressure profile can be described by:

$p_{\text {ram }}=\frac{p_{\text {max }}}{t^{2}+t_{\mathrm{HW}}^{2}}$,

where $t_{\mathrm{HW}}$ is the width of the profile (Vollmer et al. 2001). We set $p_{\max }=4000 \mathrm{~cm}^{-3}\left(\mathrm{~km} \mathrm{~s}^{-1}\right)^{2}$ and $t_{\mathrm{HW}}=50 \mathrm{Myr}$. The efficiency of ram pressure also depends on the inclination angle $i$ between the galactic disk and the orbital plane (Vollmer et al. 2001). We set $i=35^{\circ}$. The model parameters are listed in Table 4.

The large-scale evolution of NGC 4569 can be seen in Fig. 6. As expected, the stellar disk does not change, because ram pressure selectively affects only the gas. During the passage of NGC 4569 through the cluster core $(t=0$ Myr corresponds to the minimum distance between the galaxy and M 87) its gas is pushed strongly towards the south-west and finally stripped at $t>50 \mathrm{Myr}$. The gas that was pushed away, but not accelerated to the escape velocity, falls back onto the galaxy at $t>150$ Myr. This re-accretion of the gas has to be regarded with caution, since its occurence depends on the gas physics (cloud expansion, evaporation, ISM-ICM phase mixing) used in the model. We assume that the column density of the clouds does not change during the interaction - however, if it were to decrease due to, e.g., evaporation or ICM-ISM mixing, then re-accretion will be suppressed.

During the whole galaxy evolution within the cluster when ram pressure is important, only two scenarios can give rise to an asymmetric gas arm: (i) during the resettlement of the gas after a strong stripping event and (ii) during the phase of ongoing stripping. Therefore, we compare two snapshots with our H I observations: (i) the last snapshot of Fig. $6(t=300 \mathrm{Myr})$, i.e. a past stripping event and (ii) the case of ongoing stripping - another simulation made using a truncated gas disk at $R=10 \mathrm{kpc}$ with $p_{\text {max }}=2000 \mathrm{~cm}^{-3}\left(\mathrm{~km} \mathrm{~s}^{-1}\right)^{2}, i=35^{\circ}, t_{\mathrm{HW}}=80 \mathrm{Myr}$. The comparison snapshot at $t=0 \mathrm{Myr}$ is shown in Fig. 7. For a given position angle and inclination, the third angle, which is the azimuthal angle in the plane of the galaxy, has to be fixed. A variation of the azimuthal angle leads to a variation of the $3 \mathrm{D}$ direction of the galaxy's motion with respect to the observer. We chose the value that reproduces best the observed, western, low surface density arm. In both cases the galaxy moves to the north-east. We made sure that the galaxy's radial velocity did not fall below $-1500 \mathrm{~km} \mathrm{~s}^{-1}$.

We constructed model data cubes of the two snapshots and made moment maps with AIPS. Only gas particles with a local gas density greater than $100 \mathrm{~cm}^{-3}$ where put into the model cube.

\section{Comparison between simulations and observations}

In this section we compare model spectra and the three model moment maps (gas distribution, velocity field, and velocity dispersion) to those of our H I observations (Figs. 2, 3 and 5). The initial velocity field of the gas disk is shown in Fig. 8. To facilitate the comparison with the final snapshots of our simulation, we have truncated the disk at a radius of $5 \mathrm{kpc}$. Our model velocity field has a steeper rotation curve and reaches the plateau of constant rotation velocity at a smaller radius than the observed one (Fig. 3). However, the $\mathrm{CO}$ position-velocity diagram along the major axis of NGC 4569 (Fig. 23 of Kenney \& Young 1988) shows a steeper rotation curve, which is close to that of our model.

\subsection{Ongoing stripping}

The model spectra corresponding to the observed center spectrum and those of the four offset H I observations (Fig. 5) for ongoing stripping are shown in Figs. 9 and 10. The two peaks of the central double-horn spectrum have about the same velocity width and the edges of the profile are sharp. The off-center spectra in the NE, SE, and NW show maximum flux densities smaller than the detection limit of our Effelsberg data ( $\sim 5 \mathrm{mJy})$. The signature of the western arm is clearly visible at negative velocities in the $\mathrm{W}$ and $\mathrm{SW}$ spectra.

Figure 11 shows the moment maps for ongoing ram pressure stripping. As already mentioned, the projection parameters are chosen such that (i) the radial velocity of NGC 4569 and (ii) the observed, western, low surface density arm are reproduced. The central hole in the gas distribution is artificial, to avoid too small timesteps for the particle integration. The north-eastern maximum represents compressed gas that has been pushed by ram pressure to smaller galactic radii. The velocity field in the north is that of a rising rotation curve, because the ISM is pushed inwards by the ICM. This effect is also observed for NGC 4654 (Vollmer 2003). The southern part of the disk velocity field shows a plateau of constant rotation velocity. The velocity contours are continuous from the disk to the western, low surface density arm, because the gas there is accelerated and pushed out of the galaxy in the sense of rotation. As expected, the maximum of the velocity dispersion is located in the galaxy center. A second maximum can be found to the north-west. It corresponds to gas that has been pushed inwards to the north-east by the ICM and has rotated (the galaxy is rotating clock-wise) towards the west.

\subsection{Past stripping event}

The model spectra corresponding to the observed center spectrum and those of the four offset H I observations (Fig. 5) for a past stripping event are shown in Figs. 12 and 13. The low velocity peak of the central double-horn spectrum has a larger velocity width than the high velocity peak. Moreover, the lowvelocity edge of the profile shows a pronounced wing. All offcenter spectra except the one in the $\mathrm{W}$ show maximum flux 

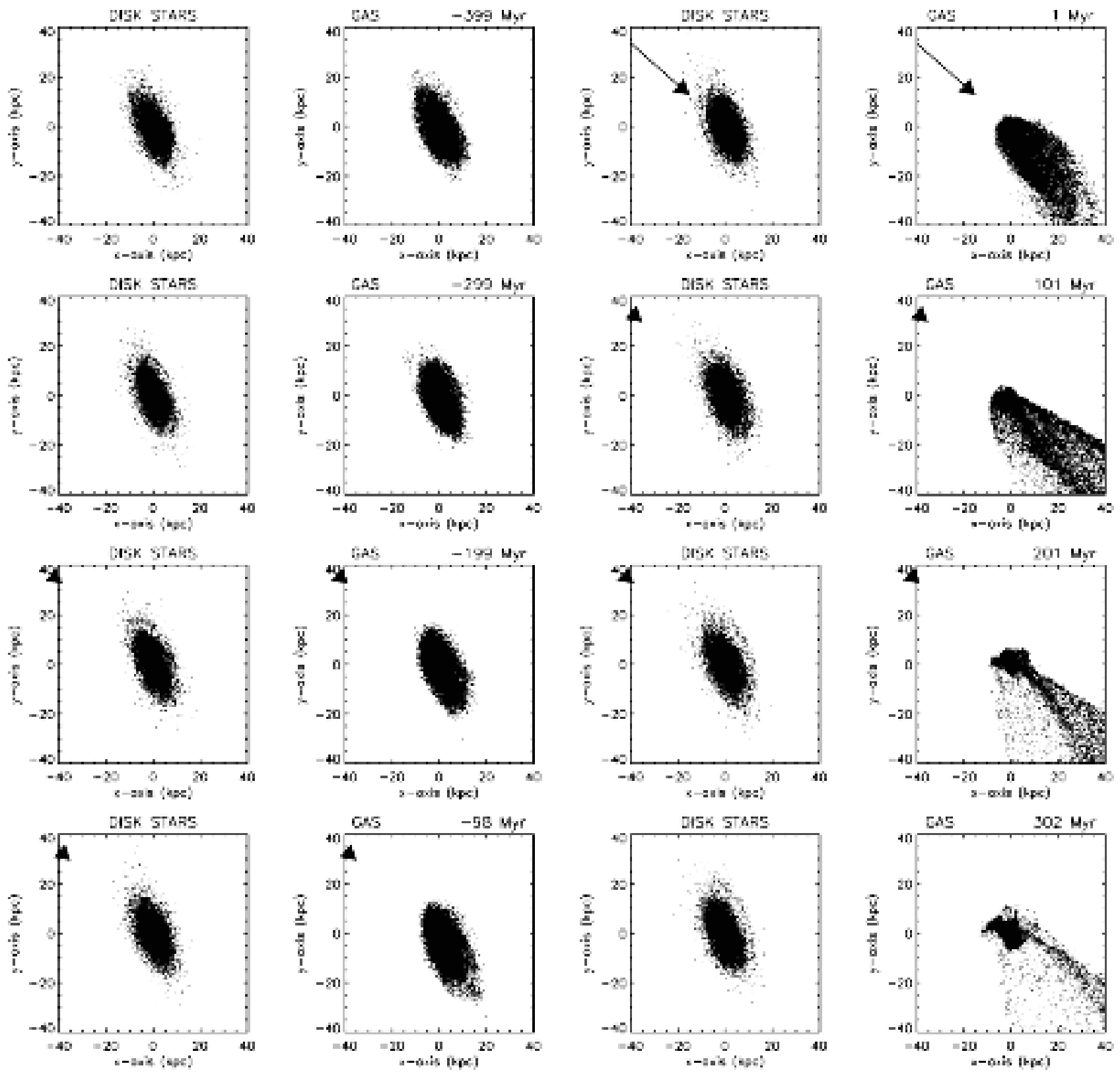

Fig. 6. Evolution of the model stellar (1st and 3rd column) and gas disk (2nd and 4th column). The major axis position angle and inclination of NGC 4569 are PA $=23^{\circ}$ and $i=62^{\circ}$, respectively. The arrow indicates the direction of ram pressure, i.e. it is opposite to the galaxy's velocity vector, and its size is proportional to $\rho v_{\text {gal }}^{2}$. The galaxy passes the cluster core at 0 Myr. The time to the core passage is marked at the top of each panel.

densities smaller than the detection limit of our Effelsberg data $(\sim 5 \mathrm{mJy})$.

Figure 14 shows the moment maps for a past ram pressure stripping event. Again, the projection parameters are chosen such that they reproduce (i) the radial velocity of NGC 4569 and (ii) the observed, western, low surface density arm. Again, the central hole in the gas distribution is artificial, to avoid too small timesteps for the particle integration. The spatial extent of the model gas distribution is smaller than that of the observed one, because we have decided to reproduce the $\mathrm{HI}$ deficiency rather than the spatial extent. For simplicity we assume that all gas in the model is atomic. Consequently, our inital central gas surface density is higher than the observed one (our model rather represents an $\mathrm{Sc} / \mathrm{Sb}$ galaxy, whereas NGC 4569 is classified as Sab). Thus, for a given H I deficiency, our model gas disk is smaller than the observed disk. The disk velocity field shows a plateau on both sides, which is reached at smaller galactic radii in the south than in the north. The velocity field of the southern part of the western, low surface density arm is not a continuation of that of the disk. The absolute velocities of the arm are higher than those of the neighbouring disk. The maximum of the velocity dispersion is located in the center. A second, point-like maximum can be found to the south-east of the nucleus where the radial velocity has its minimum value. This feature is due to a gas streamer that falls onto the galaxy from behind. In addition, we observe an extended 

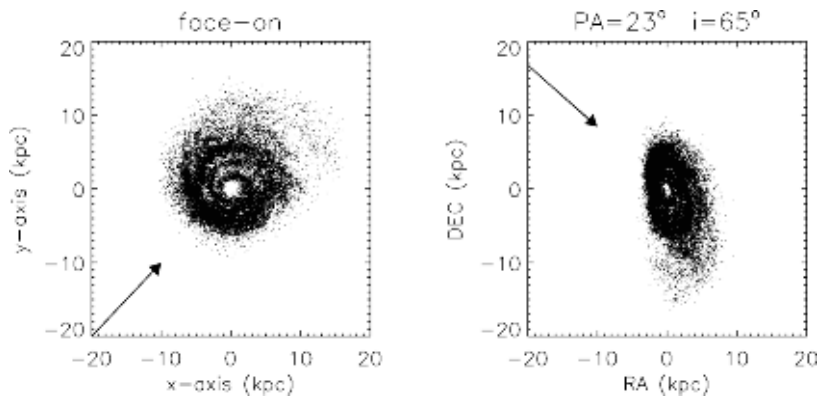

Fig. 7. Comparison snapshot of the gas distribution of NGC 4569 for the case of ongoing ram pressure stripping. Left panel: the disk seen face-on. Right panel: view of the disk using the observed position angle and inclination of NGC 4569. The arrow indicates the direction of the wind, i.e. opposite to the galaxy's motion.

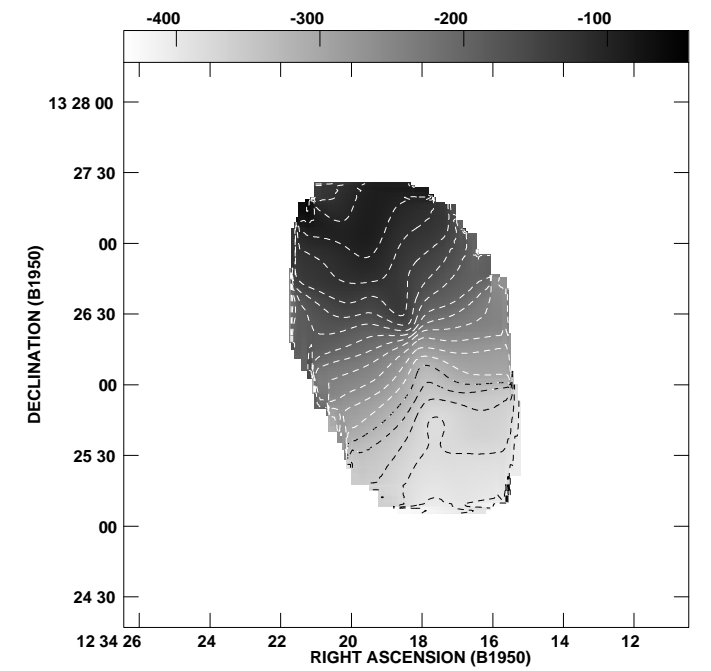

Fig. 8. Initial gas velocity field. The disk is truncated at a radius of $5 \mathrm{kpc}$.

maximum at the inner edge of the western, low surface density arm south of the nucleus.

\section{Discussion}

Since both models reproduce the observed western, low surface density arm as well as the radial velocity of NGC 4569, it became clear that it is impossible to chose between the two scenarios only on the basis of the observed gas distribution. Only the velocity information in addition to the gas distribution allows us to discriminate between the models.

The extended low velocity edge of the observed central H I spectrum is only reproduced by the past stripping simulation. In general, the offset spectra of both simulations are consistent with our observations, i.e. only in the west (W) is atomic gas detected (flux density greater than $\sim 5 \mathrm{mJy}$ in one $20 \mathrm{~km} \mathrm{~s}^{-1}$ channel). The western arm of the ongoing stripping model is somewhat too prominent in the model SW spectrum, but this is due to the detailed choice of the stripping parameters. It is important to note that the tail of stripped and back-falling gas of the post-stripping model (Fig. 6) could not have been detected with the Effelberg telescope (Fig. 5) neither with the VLA.

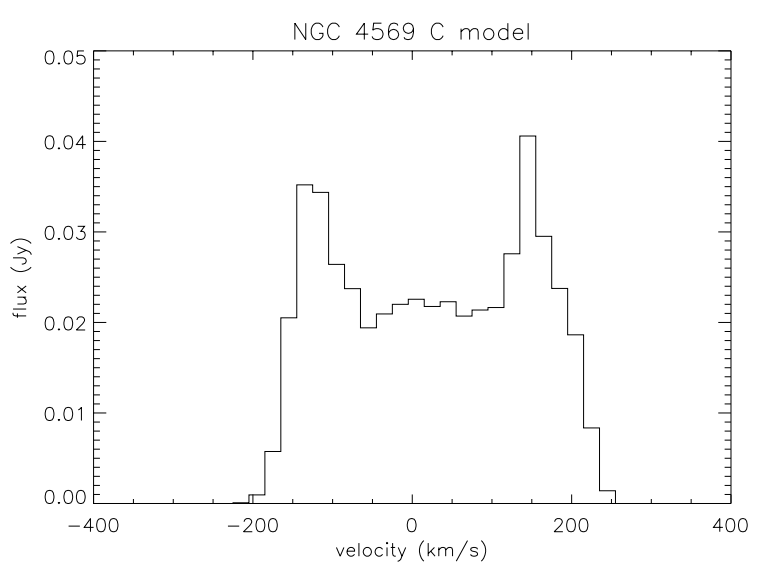

Fig. 9. Model H I spectrum for ongoing ram pressure stripping.
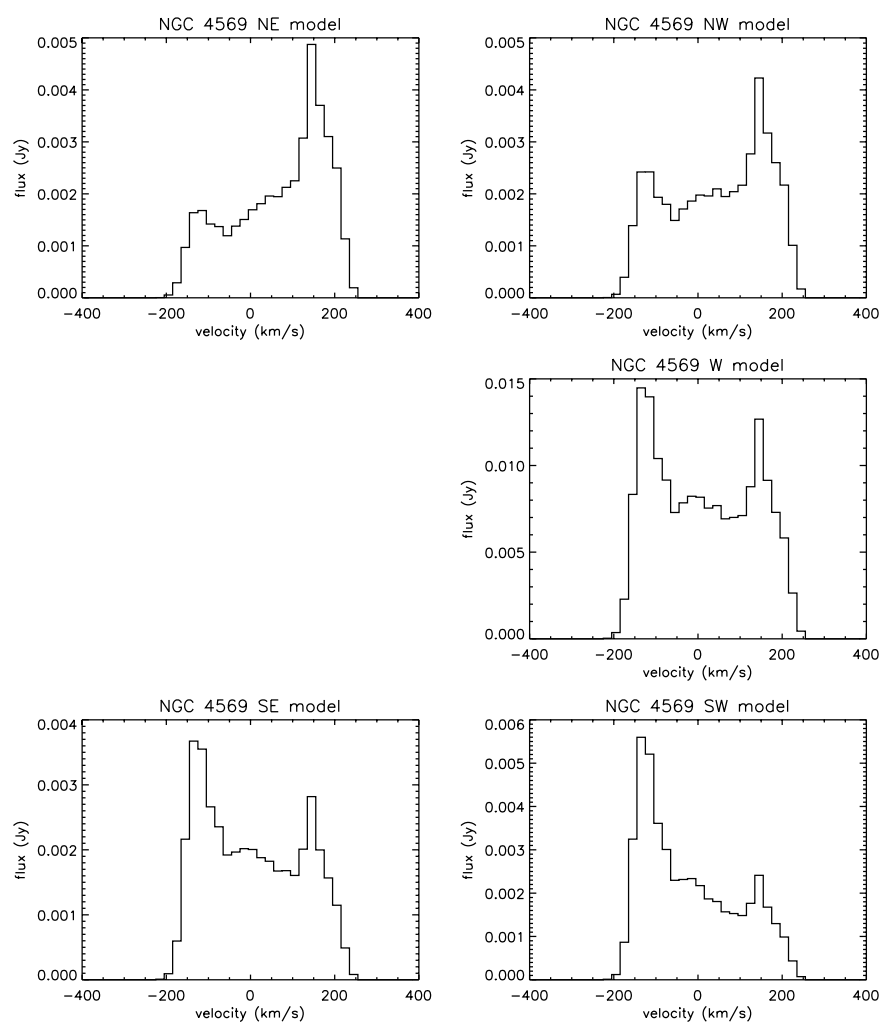

Fig. 10. Model spectra corresponding to our offset $\mathrm{H}$ I observations (Fig. 5) for ongoing stripping.

Ongoing stripping steepens the velocity field in the direction of the galaxy's motion and elongates the velocity contours on the opposite side (Fig. 11; see also Vollmer 2003), because ram pressure pushes the gas to smaller galactic radii in the direction of the galaxy's motion and accelerates it in the sense of rotation on the other side. On the other hand, a past stripping event causes the steepening and asymmetry of the rotation curve (Fig. 14). This asymmetry is also seen in the observed velocity field (Fig. 3) where the rotation curve of the southern half is steeper than that in the north. Regarding the general steepening of the rotation curve: since we do not know the initial velocity field of NGC 4569, we do not think that the difference between the simulated (Fig. 14) and observed (Fig. 3) velocity fields poses a problem for the validity of the simulations. 

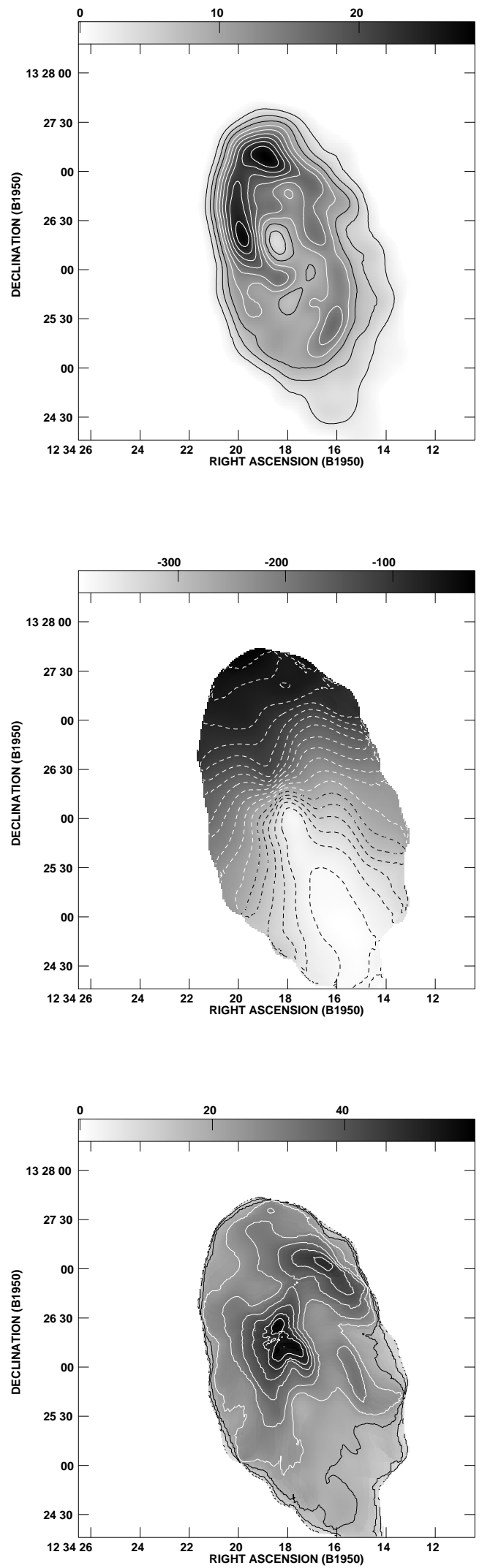

Fig. 11. Model moment maps for ongoing ram pressure stripping. Upper panel: gas distribution in arbitrary units. The relative contours are the same as in Fig. 3. Middle panel: velocity field. The contours are the same as in Fig. 3. Lower panel: velocity dispersion. The contours are the same as in Fig. 3.

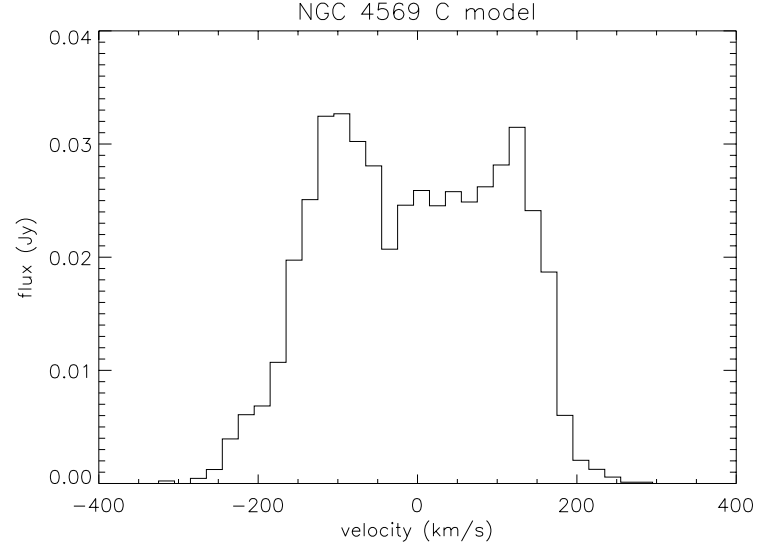

Fig. 12. Model H I spectrum for a past ram pressure stripping event.
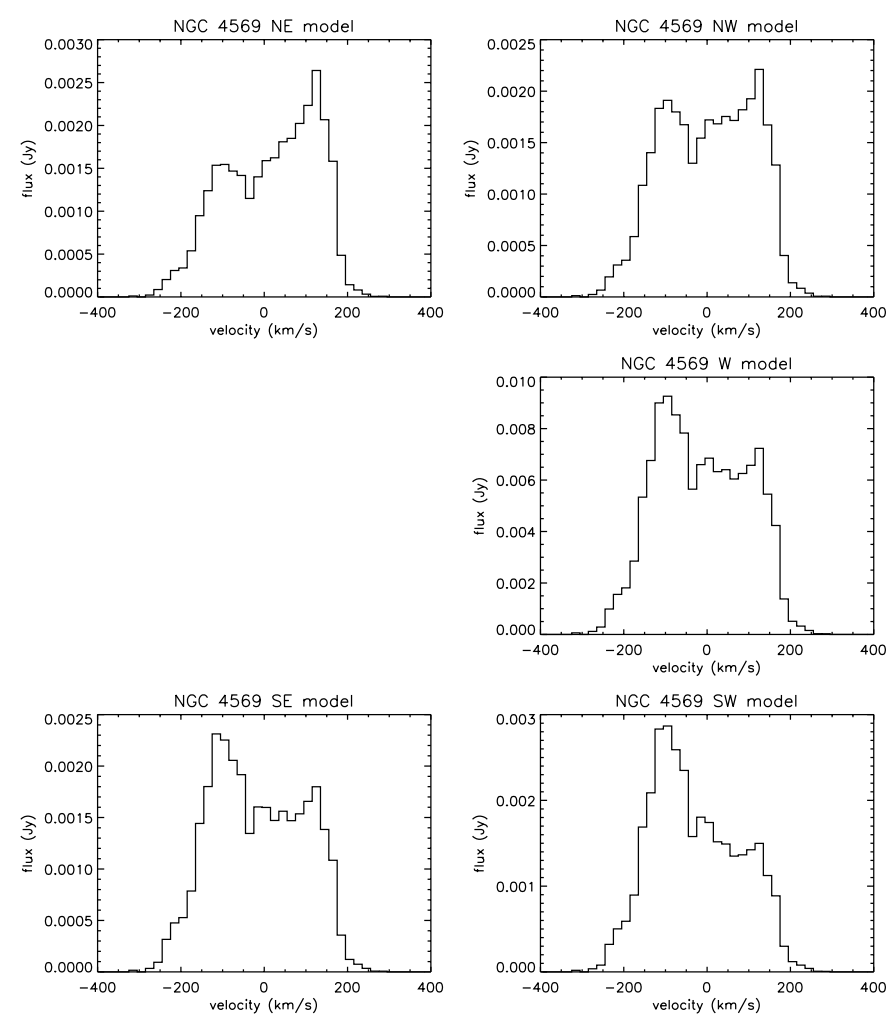

Fig. 13. Model spectra corresponding to our offset H I observations (Fig. 5) for a past ram pressure stripping event.

Whereas the velocity field in the western arm region is continuous in the case of ongoing stripping, it shows a discontinuity in the post-stripping scenario as is observed. Also, the difference in absolute velocity between the disk and the western arm of the post-stripping scenario fits nicely our H I observations. For the distribution of the velocity dispersion, the observed, single, local, extended maximum at the inner edge of the western am is only reproduced by the post-stripping model. It is located at the interface between the western, low surface density arm and the gas rotating within the galactic disk. We failed to find any suspect features in the 3D gas distribution at that location. The general dynamics are very complicated, which makes it hard to disentangle different physical effects due re-accretion, gas expansion, and rotation. The lines in this region are 

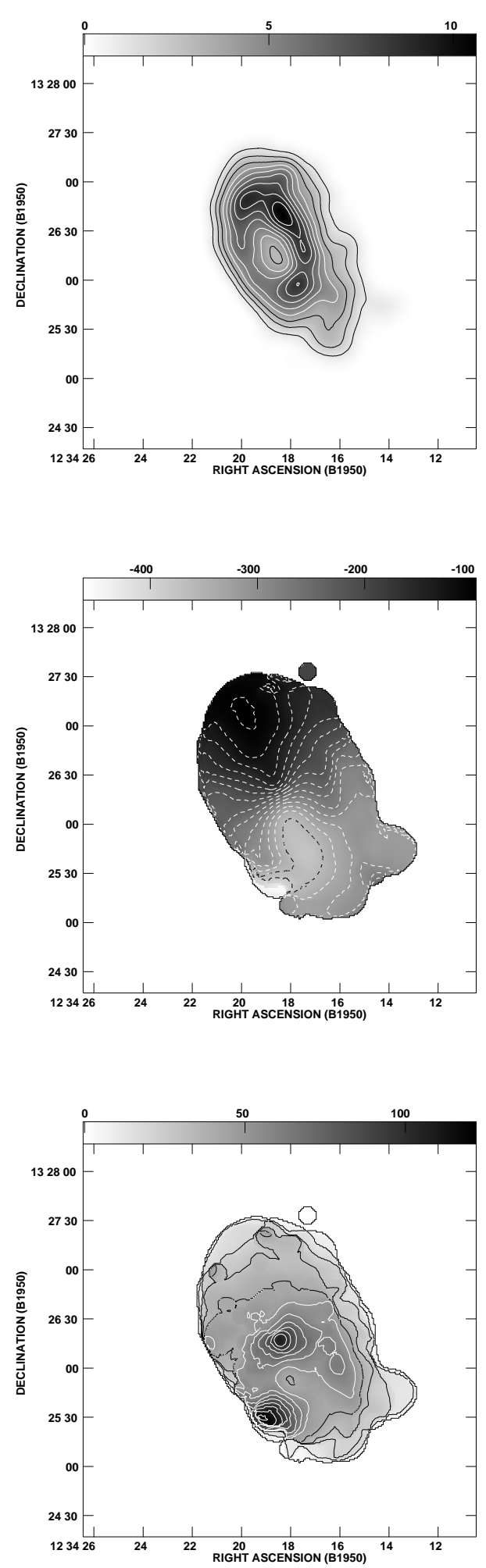

Fig. 14. Model moment maps for a past ram pressure stripping event. Upper panel: gas distribution in arbitrary units. The relative contours are the same as in Fig. 3. Middle panel: velocity field. The contours are the same as in Fig. 3. Lower panel: velocity dispersion. The contours are the same as in Fig. 3.

broadened to both larger and smaller radial velocities compared to a region south of it. We have no clear explanation for why this region shows an enhanced velocity dispersion.
There is also a point-like maximum visible, like the one observed, but it is located to the south. The enhanced linewidth arises from a back-falling gas streamer. Since the exact dynamics of such a streamer depend on gas physics (e.g. the volume filling factor, shock heating, evaporation) and the detailed stripping parameters, we do not expect to fit its exact location. We can only speculate that such a region of unusually high velocity dispersion might be a sign of re-accretion.

We thus conclude that the ongoing stripping scenario is ruled out based on the comparison of the observed and simulated velocity fields and velocity dispersion distributions. On the other hand, the post-stripping scenario reproduces the major characteristics of the observations: the truncated gas disk, the western, low surface density arm, and the discontinuity between the velocity field in the galactic disc and the western arm. In order to investigate if the gas disk of NGC 4569 was already truncated before it underwent the ram pressure stripping event that lead to the observed H I deficiency, we made an additional simulation starting with a truncated gas disk at $R=10 \mathrm{kpc}$. The resulting moment maps are not distinguishable from that of Fig. 14. Thus we conclude that it is not excluded that NGC 4569 had already experienced a ram pressure event more than $\sim 1$ Gyr ago that lead to a truncated H I disk. After such a first ram pressure stripping event the gas disk had enough time to evolve into an equilibrium state without major perturbations. Possibly, the gas disk became more robust via the "disk annealing process" described by Schulz \& Struck (2001). This is consistent with our finding that the rotation curve steepens after the ram pressure event. During the galaxy's motion through the outer parts of the cluster some of its ISM might have been replenished by the gas released by dying stars. All we can say is that NGC 4569 had a less truncated gas disk before the last ram pressure stripping event than it has now. For each stripping event, the following parameters are important for the efficiency of ram pressure stripping: the gas disk truncation radius, the galaxy orbit, and the inclination angle between the galaxy's disk and the orbital plane during the cluster core passage. Different core passages can have different maximum ram pressures (Vollmer et al. 2001), and the one with the highest maximum ram pressure is decisive for the galaxy. NGC 4569 experienced its most efficient ram pressure stripping event $\sim 300$ Myr ago.

A final comparison of the data cubes can be directly made in 3 dimensional representations. We have developed a 3D visualisation of data cubes that enables us to easily distinguish different kinematic features (see Vollmer et al. 1999). All velocity channels seen in Fig. 1 are piled up to give the cube whose axes are the right ascension, the declination and the radial velocity. All points in the cube having intensities exceeding a chosen level ( $2 \mathrm{mJy} / \mathrm{beam})$ become opaque, the rest remain transparent. The surface created in this way is illuminated by light which is coming from the observer's direction, making features that are located closer to the observer brigther. If we had, for example, a sphere of intensity increasing to the centre, it would appear as a ball with its radius increasing with decreasing chosen intensity level. This representation allows to analyse the whole cube from any possible point of view. In Fig. 15 we have rotated the cube so that the RA-Dec plane 

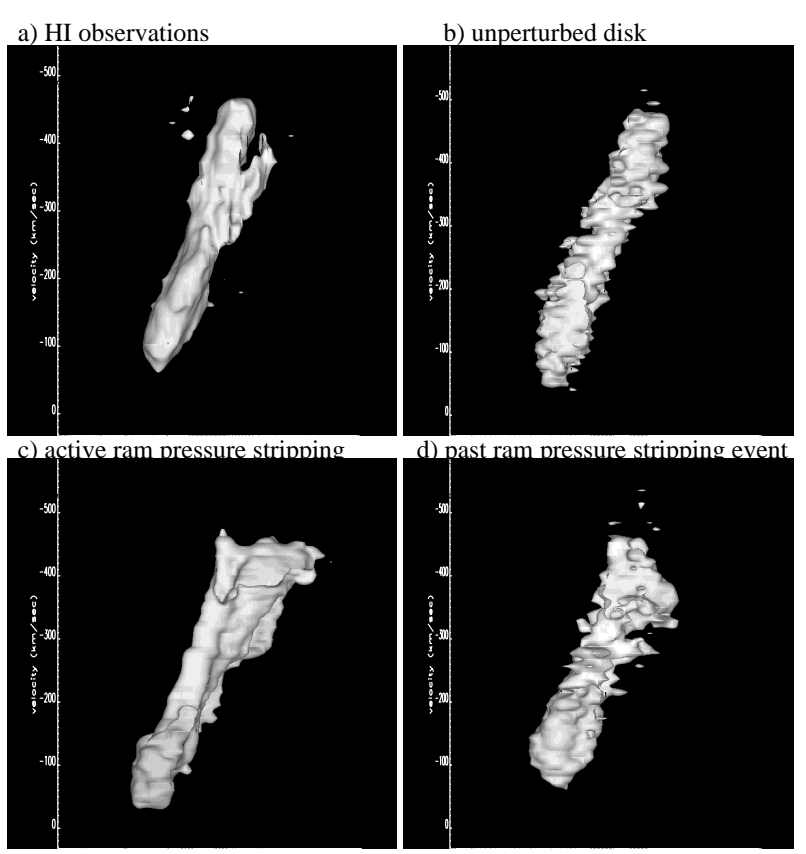

Fig. 15. Three dimensional view of the data cubes. The RA-Dec plane is perpendicular to the image plane, and the velocity axis is the vertical axis. a) H I observations. b) Unperturbed gas disk. c) Ongoing ram pressure stripping. d) Past ram pressure stripping event.

is perpendicular to the image plane, and the velocity axis is the vertical axis. The kinematics of rotation form a disk-like structure in the restricted phase space (RA, Dec, $v_{\mathrm{r}}$ ). We have rotated the cube such that this disk structure is seen edge-on. The observed western, low surface density arm can be seen in the upper right part of the image and is clearly distinct from the galaxy rotation (Fig. 15a). For comparison we show the unperturbed gas disk in Fig. 15b. The western arm in the ongoing stripping scenario (Fig. 15c) is spatially more extended and extends to smaller velocities. That in the post-stripping scenario (Fig. 15d) nicely fits the 3 dimensional extent of the observed western arm. However, due to the southern plateau of the model velocity field, the southern part of the disk-like structure in the restricted phase space is thicker than the observed one. We thus conclude that the post-stripping scenario is the most probable one for NGC 4569.

This galaxy is not the first one where an extended, low surface density arm has been discovered in deep $\mathrm{H}$ I imaging observations as an extended, low surface density arm has also been found in three other Virgo spiral galaxies: NGC 4321 (Knapen et al. 1993), NGC 4654 (Phookun \& Mundy 1995) and NGC 4548 (Vollmer et al. 1999). An extreme case is NGC 4388 where Vollmer \& Huchtmeier (2003) detected neutral hydrogen more than $20 \mathrm{kpc}$ above the disk plane. These low surface density features are in contrast to high surface brightness, extraplanar emission regions located close to the disk. These have been observed in NGC 4522 (Kenney et al. 2003) and NGC 4438 (Cayatte et al. 1990), two galaxies that are most probably actively stripped.

The question arises whether the high and low surface density features are caused by two different stages of an ICM-ISM interaction. We might speculate that the low surface brightness features whose velocity fields are not a continuation of that of the neighbouring disk are reminiscent of a past stripping event (NGC 4569 and NGC 4548), whereas those with a continuous velocity field are due to weak ongoing ram pressure stripping, perhaps in addition to a gravitational interaction (NGC 4654). On the other hand, high surface brightness features appear to be evidence of a major ongoing stripping process.

If we assume a temporal ram pressure profile as presented in Vollmer et al. (2001) the observational groups might be understood in the following way:

(i) strong stripping leading to a large $\mathrm{HI}$ deficiency (NGC 4388, NGC 4438, NGC 4522, NGC 4548, NGC 4569): during the maximum ram pressure phase high surface density gas is pushed out of the galactic disk (NGC 4438, NGC 4522). When the pushed-out gas moves to greater distances from the galaxy it expands and evaporates, leading to low surface density H I (NGC 4388). During active ram pressure stripping, high surface density gas is accelerated and pushed to smaller galactic radii in the direction of the galaxy's motion and to larger galactic radii in the opposite direction. When the galaxy leaves the cluster core, the ram pressure decreases strongly and the gas is left with just the gravitational potential of the galaxy, to which it tries to adapt itself. The compressed region expands and the pushed-away gas falls back to the galaxy. This creates expanding, asymmetric ring structures whose surface density decreases during their expansion. These short-lived $\left(<10^{8} \mathrm{yr}\right)$ features represent the kinetically perturbed low surface density arms observed in NGC 4569 (and maybe NGC 4321 and NGC 4548, whose cases are less clear). The number of observed low surface density arms in Virgo cluster spirals is small, but since these observations are time consuming, only a few galaxies have been observed deeply enough to allow their detection. However, all observations of sufficient depth have shown the presence of a low surface density arm;

(ii) weak stripping together with a gravitational interaction leading to a small or even negligible H I deficiency: ram pressure pushes away the low surface density gas which has been torn out of the galactic disk by the gravitational interaction (NGC 4654).

This hypothesis needs to be verified with a larger sample of cluster spirals.

\section{Conclusions}

We obtained deep H I line observations of the anemic Virgo spiral galaxy NGC 4569 with the VLA in its D configuration and with the Effelsberg 100-m telescope. Snapshots of a dynamical model including the effects of ram pressure were compared to the observations. Two different stages of a ram pressure stripping event were investigated: (i) ongoing stripping and (ii) a past stripping event. The results of our investigations are:

1. We detected a low surface density arm in the west of the galaxy. 
2. The velocity field of this arm is not a continuation of the velocity field of disk rotation.

3. The absolute radial velocities of the arm are higher than those of the neighbouring disk.

4. The velocity dispersion shows a local maximum at the inner edge of the arm.

5. There is no gas mass greater than $10^{7} M_{\odot}$ detected far away $(>20 \mathrm{kpc})$ from the galaxy.

6. The post-stripping scenario can reproduce the main characteristics of the gas distribution, velocity field, and velocity dispersion together with the radial velocity of the galaxy.

7. Within this scenario the galaxy passed through the cluster core about 300 Myr ago.

8. It is not excluded that NGC 4569 had an already truncated gas disk before this ICM-ISM interaction.

Acknowledgements. Based on observations with the 100-m telescope of the MPIfR (Max-Planck-Institut für Radioastronomie) at Effelsberg. The Very Large Array of the National Radio Astronomy Observatory (NRAO) is operated by Associated Universities Inc., under contract with the National Science Foundation.

\section{References}

Bottinelli, L., Gouguenheim, L., \& Paturel, G. 1983, A\&A, 118, 4 Boulares, A., \& Cox, D. P. 1990, ApJ, 365, 544

Cayatte, V., van Gorkom, J. H., Balkowski, C., \& Kotanyi, C. 1990, AJ, 100, 604

Cayatte, V., Kotanyi, C., Balkowski, C., \& van Gorkom, J. H. 1994, AJ, 107, 1003 de Vaucouleurs, G., de Vaucouleurs, A., Corwin, H. G., et al. 1991, Third Reference Catalogue of Bright Galaxies (New York: Springer) (RC3)

Elmegreen, B. G., \& Falgaron, E. 1996, ApJ, 471, 816

Guharthakurta, P., van Gorkom, J. H., Kotanyi, C. G., \& Balkowski, C. 1988, AJ, 96, 851

Keel, W. C. 1996, PASP, 108, 917

Kenney, J. D., \& Young, J. S. 1988, ApJS, 66, 261

Kenney, J. D., van Gorkom, J. H., \& Vollmer, B. 2003, in preparation

Knapen, J. H., Cepa, J., Beckman, J. E., Soledad del Rio, M., \& Pedlar, A. 1993, ApJ, 416, 563

Napier, P. J., Thompson, A. R., \& Ekers, R. D. 1983, Proc. IEEE, 71, 1295

Phookun, B., \& Mundy, L. G. 1995, ApJ, 453, 154

Schulz, S., \& Struck, C. 2001, MNRAS, 328, 185

Solanes, J. M., Manrique, A., Garcia-Gomez, C., et al. 2001, ApJ, 548, 97

Springel, V., Yoshida, N., \& White, D. M. 2001, NA, 6, 79

Tschöke, D., Bomans, D. J., Hensler, G., \& Junkes, N. 2001, A\&A, 380,40

van den Bergh, S. 1976, ApJ, 206, 883

Vollmer, B., Cayatte, V., Boselli, A., Balkowski, C., \& Duschl, W. J. 1999, A\&A, 349, 411

Vollmer, B., Cayatte, V., Balkowski, C., \& Duschl, W. J. 2001, ApJ, 561,708

Vollmer, B., \& Huchtmeier, W. 2003, A\&A, 406, 427

Vollmer, B. 2003, A\&A, 398, 525

Warmels, R. H. 1988, A\&AS, 72, 57

Wiegel, W. 1994, Diploma Thesis, University of Heidelberg 